This is a postprint version of the following published document:

Experimental response of agglomerated cork under multi-impact loads S. Sanchez-Saez, E. Barbero, S.K. Garcia-Castillo, I. Ivañez, J. Cirne Materials Letters 160 (2015) 1 december, pp. 327-330.

Available http://dx.doi.org/10.1016/j.matlet.2015.08.012.

(C) 2005 Elsevier

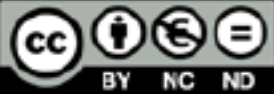

This work is licensed under a Creative Commons Attribution-NonCommercialNoDerivatives 4.0 International License. 


\title{
Experimental response of agglomerated cork under multi-impact loads
}

\author{
S. Sanchez-Saez ${ }^{\text {a,* }}$, E. Barbero ${ }^{\text {a }}$, S.K. Garcia-Castillo ${ }^{\text {a }}$, I. Ivañez ${ }^{\text {a }}$, J. Cirne ${ }^{\text {b }}$ \\ a Department of Continuum Mechanics and Structural Analysis, University Carlos III of Madrid, Avenida de la Universidad 30, 28911 Leganés, Madrid, \\ Spain ${ }^{\mathrm{b}}$ Structural Integrity Group, Mechanical Engineering Department, University of Coimbra, 3030-788 Coimbra, Portugal
}

\begin{abstract}
In contrast to other cellular materials, which deform by crushing under impact and develop permanent deformation, agglomerated cork has a viscoelastic response and is environmentally friendly; therefore, it could be a good alternative to be used in engineering applications subjected to more than one impact, and thus further understanding of the energy-absorption capabilities of this material under multi-impact conditions is needed. In this work the multiimpact behaviour of agglomerated cork was studied ex-perimentally by performing several consecutive impacts in a drop-weight tower on specimens of dif-ferent thickness and at two impact energy levels. The maximum contact force, maximum strain, and the absorbed energy were evaluated in each test. The results show the great capability of agglomerated cork to continue absorbing energy after several consecutive impacts.
\end{abstract}

Keywords: Agglomerated cork, Impact behaviour, Natural material, Multi-impact applications.

\section{Introduction}

Due to increasing environmental consciousness and the need for sustainable development, the use of natural materials in engineering applications has increased during recent years, as these materials are obtained from renewable resources and they facilitate recycling the components at the end of their service life.

Agglomerated cork is a highly durable cellular material which is ecological and hygienic, can be recycled and reused and has easy maintenance [1]. It is made from a mixture of natural cork and an organic binder that is pressed in an autoclave and crossed by a water-vapour flow at high temperatures. It has some remarkable properties, such as high damage tolerance to impact loads, good thermal and acoustic insulation and excellent damping characteristics for the suppression of vibrations.

Cellular materials are widely used in road-safety applications [2-5], or in packaging applications [6] for the protection of objects/ products. Normally, in these applications, the components are rarely subjected to a single impact. Under impact, the majority of the cellular materials deform by crushing, reaching high strains and developing permanent deformation. This means that after the first impact, the energy-absorption capacity of these materials is reduced almost to zero. However, agglomerated cork has a viscoelastic behaviour, deforming mainly elastically. For this reason, agglomerated cork could be a good alternative in applications subject to more than one impact.

\footnotetext{
* Corresponding author.

E-mail address: ssanchez@ing.uc3m.es (S. Sanchez-Saez).
}

Research on the mechanical behaviour of agglomerated cork under impact loads is limited to a small number of studies related to low-velocity impact tests in a drop tower [7-9], dynamic crushing behaviour [10], and ballistic behaviour of agglomerated cork [11]. However, information is still lacking on the multi-impact behaviour of agglomerated cork.

The aim of the present work is to analyse the behaviour of agglomerated cork subjected to several consecutive impacts. Specimens with different thicknesses were subjected to two impact energies. The maximum force, maximum strain, and the absorbed energy were analysed.

\section{Material and experimental procedure}

The material used was a commercial agglomerated cork manufactured by Amorin Cork Composites (NL-10). This agglomerated cork was made from a mixture of cork particles and a polymeric binder, with a density of $140 \mathrm{~kg} / \mathrm{m}^{3}$.

Agglomerated cork follows the typical crushing behaviour of cellular materials. Fig. 1a plots the compression load-strain curve of a static test performed by the authors, using a cross-head speed of $0.5 \mathrm{~mm} / \mathrm{min}$. The curve is divided into three main regions: elastic, plateau, and densification regions. The plateau region is not perfectly horizontal because force continues increasing during cell collapse, due to the heterogeneity of the structure, forming a barrier to overcome [12].

Low-velocity impact tests were performed using an in-strumented drop-weight tower CEAST Fractovis 6785 (Fig. 1b). 
a

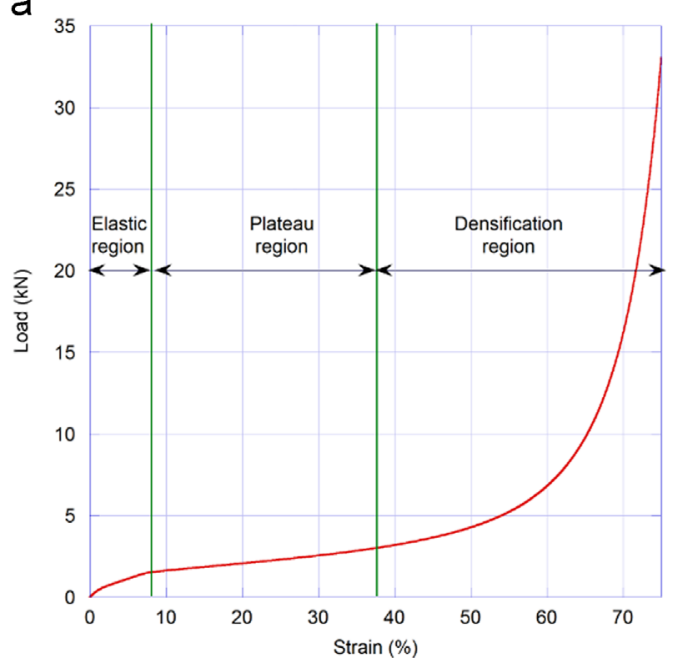

b

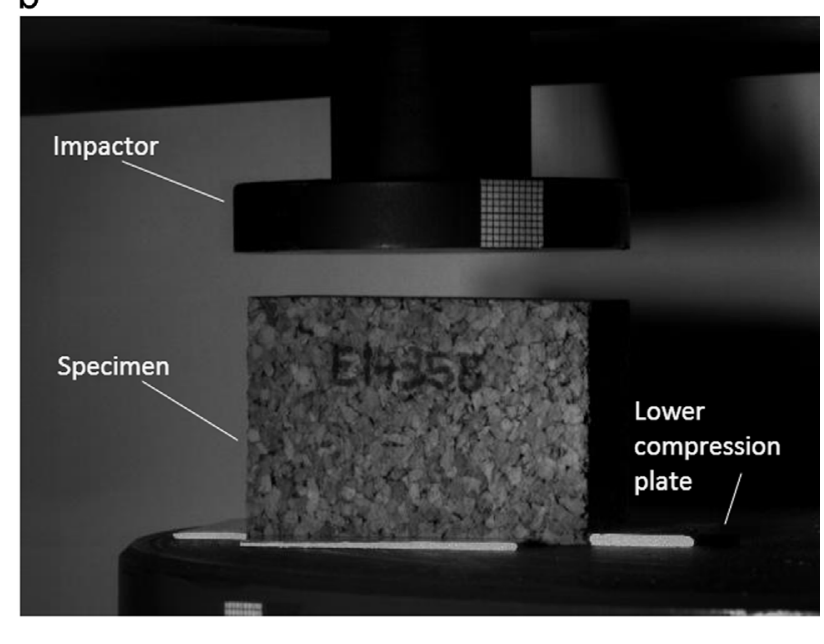

Fig. 1. (a) Compression load-strain curve for NL-10 agglomerated cork, and (b) low-velocity impact test of a 35 mm thick agglomerated-cork specimen.

Three consecutive impacts were performed for each specimen with time differences of $1.5 \mathrm{~min}$ (1st, 2nd, and 3rd impacts). The impactor had a circular-flat tip and a total mass of $4.134 \mathrm{~kg}$. Specimens had a cross-section of $50 \mathrm{~mm} \times 50 \mathrm{~mm}$ and three different thicknesses (35, 50, and $70 \mathrm{~mm}$ ). Two impact energies were used in the tests: $17.5 \mathrm{~J}$ and $35 \mathrm{~J}$. A total of 36 tests were performed. In all tests, the specimens were centred on a lower compression plate.

Each test provided a record of the load applied to the specimen by the impactor, and was recorded by a high-speed video camera APX PHOTRON FASTCAM to measure the impact velocity, the displacement of the specimen and the energy absorbed by the specimen.

\section{Results}

The maximum strain of the specimen, the maximum contact force between the impactor and specimen, and the absorbed energy in the crushing process were determined for all the impact tests carried out. Despite the natural character of the material, a relatively low scatter was observed: $7.5 \%$ for the maximum strain and force, and $9 \%$ for the absorbed energy.

\subsection{Maximum strain of the specimen}

Two impact-energy levels were considered for the consecutive impact tests. Fig. 2 displays the maximum strain reached for each case, which increased with the impact-energy level. In addition, this value increased with each consecutive test.

At an impact-energy level of $17.5 \mathrm{~J}$, the increment in strain between 1 st and 3rd impact was similar in both 50-mm-thick and 70 -mm-thick specimens, being $13 \%$ and $16 \%$, respectively. At impact energy of $35 \mathrm{~J}$, the increment between 1st and 3rd consecutive impact for the same specimens was almost the same, $20 \%$ and $21 \%$, respectively. In both cases, the maximum strains reached corresponded to a strain level clearly located in the plateau region of the load-strain curve shown in Fig. 1a.

For geometrical reasons, the $35 \mathrm{~mm}$-thick specimens presented the highest strain values for the same impact-energy levels (both high and low) when compared to thicker specimens. The increment in strain for the lowest impact energy tested, between $1 \mathrm{st}$ and 3 rd impact, was $31 \%$, i.e. twice the value observed in thicker specimens. At an impact energy of $17.5 \mathrm{~J}$, the maximum strain

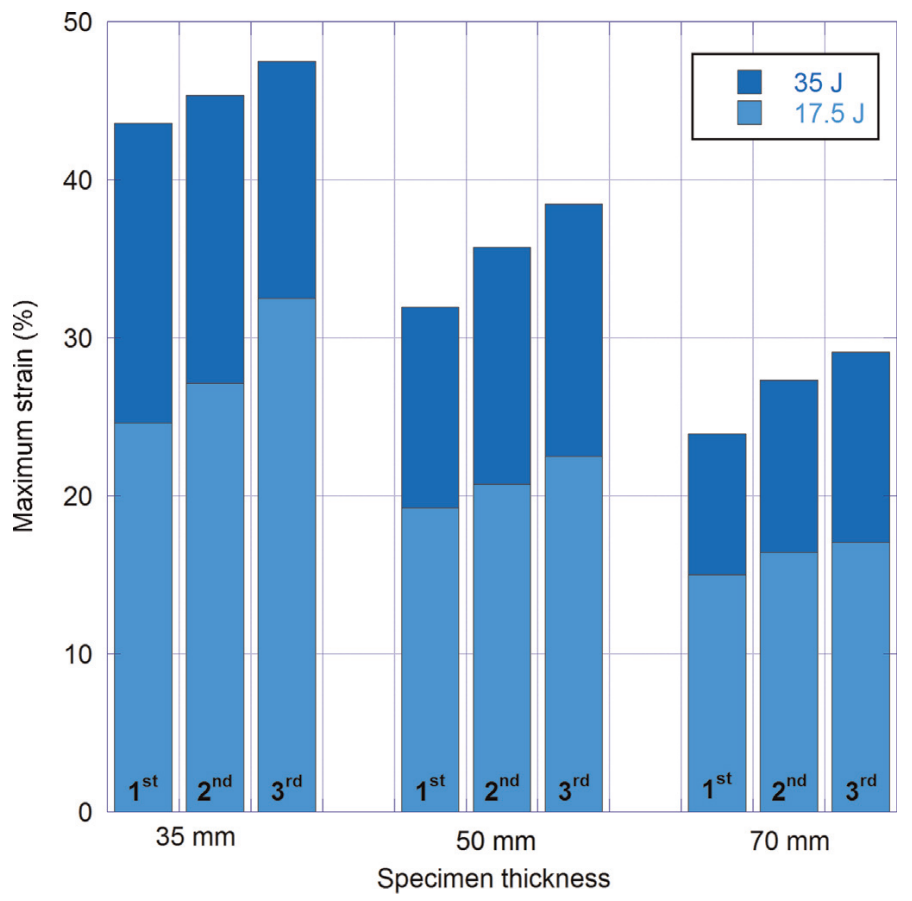

Fig. 2. Maximum strain of the specimens.

values were around $25-31 \%$, still located in the plateau stage in the load-strain curve shown in Fig. 1a.

However, for an impact energy level of $35 \mathrm{~J}$, the maximum strain reached in these specimens was $42.7 \%$ at the 1 st impact, and $47.5 \%$ at the 3rd impact. Both maximum strain values corresponded to strain levels located at the onset of the densification stage. For this reason, at impact energy of $35 \mathrm{~J}$, the consecutive impacts on 35-mm-thick specimens, increased the maximum strain by only $9 \%$, between 1 st and 3rd impact, this being similar to the scatter observed in the experimental tests for this variable (7.5\%). This means that for 35-mm-thick specimens, the increment in strain at the highest impact energy tested was almost negligible, as the agglomerated cork reached the densification stage and became densified, decreasing the capability of deformation. Thicker specimens showed maximum strain levels of around $25-30 \%$ at an impact energy of $35 \mathrm{~J}$, and thus no specimen reached the densification region, allowing the increase of this variable. 


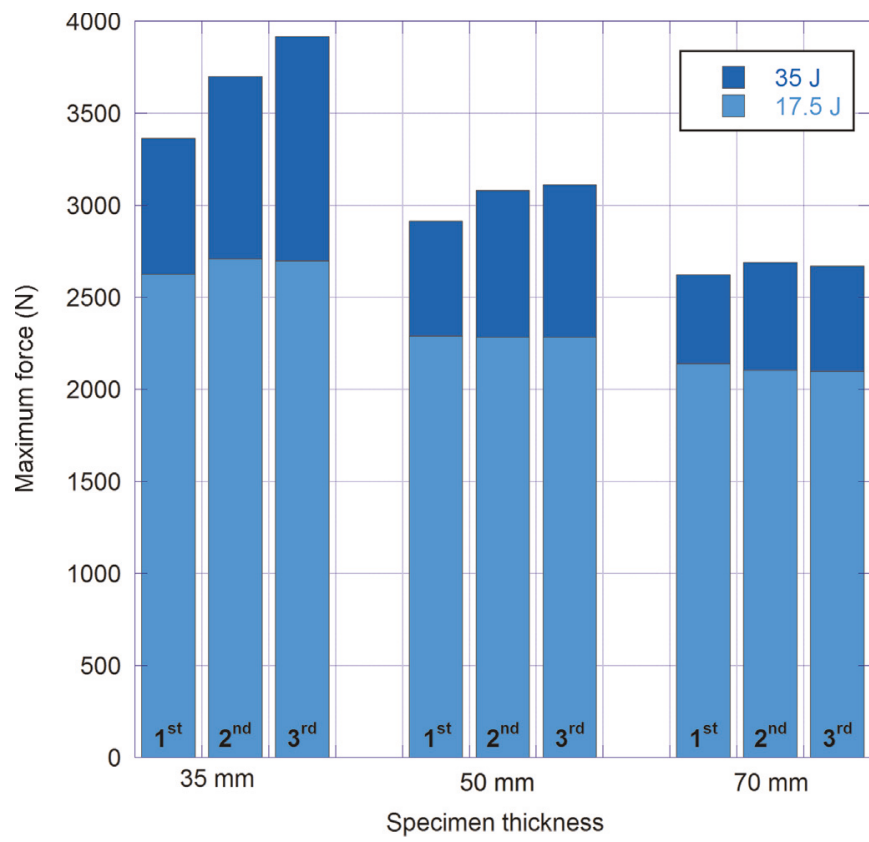

Fig. 3. Maximum contact force between the impactor and the specimen.

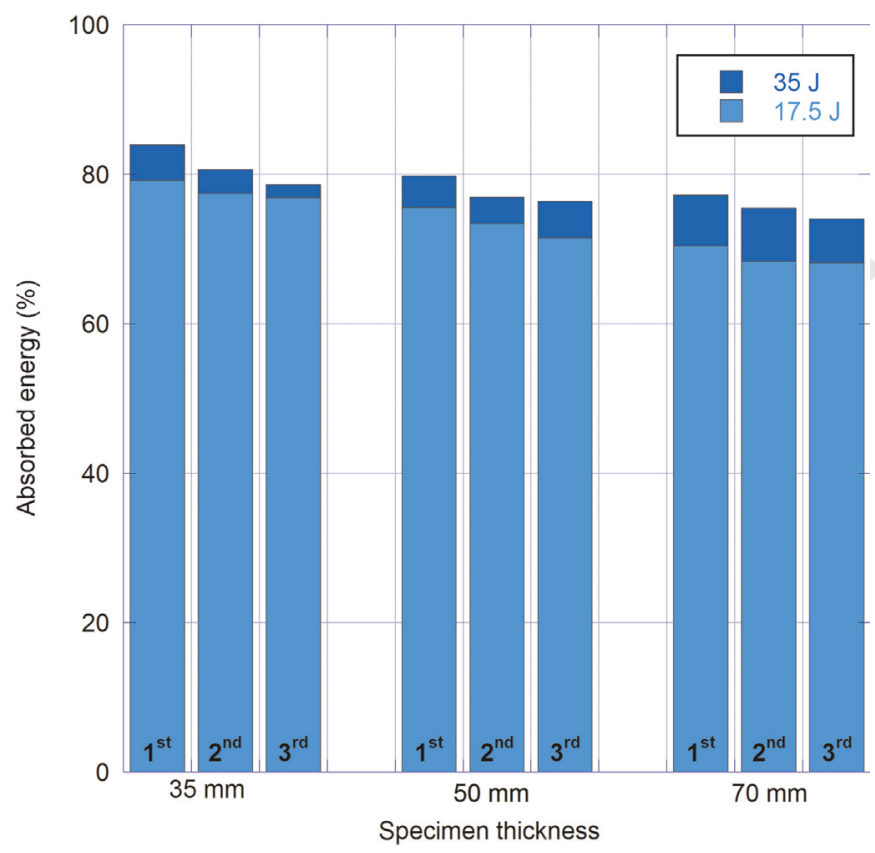

Fig. 4. Percentage of absorbed energy by the agglomerated-cork specimens.

\subsection{Maximum contact force}

Fig. 3 represents the maximum contact force during the multiimpact tests (1st, 2nd, and 3rd impacts) for each specimen thickness, impacted at the two different impact-energy levels studied. The maximum force value and trend can be divided into two different behaviours, depending on the impact-energy level, as they present certain differences.

For the lowest impact-energy level $(17.5 \mathrm{~J})$, the maximum contact force remains almost constant for all specimen thicknesses, and consecutive impact. However, at higher energy levels, for specimens $35 \mathrm{~mm}$ thick, the maximum contact force increased with each impact event (16\% between the first and the third impact). As shown in the previous section, this behaviour can be related to the densification undergone by agglomerated cork as it is compressed during the repeated impact events. At higher energy levels, the cork specimen is more compressed, and reaches the densification stage at which the load increases rapidly with the strain, Fig. 1a.

For the thicker specimens, the maximum strains are located in the plateau region, and the maximum contact force remains almost constant, as can be seen in Fig. 1a. For the $50-$ mm-thick specimens, a slight increase of $6 \%$ appeared between first and second impact at $35 \mathrm{~J}$, but this increment was lower than the scatter detected (7\%) and thus can be considered negligible.

To find a noticeable increment in maximum contact force in consecutive impacts, higher impact energies than $35 \mathrm{~J}$ should be applied in the thicker specimens.

\subsection{Absorbed energy}

Fig. 4 shows the energy-absorption percentage for each specimen at each consecutive impact. A high percentage of the impact energy was absorbed (70-80\%) for both energy levels. The absorbed-energy percentage increased slightly with the impact-energy level, i.e. around $10 \%$.

These results show the excellent energy-absorption properties of cork material: the energy absorption was considerable after every impact. The absorbed energy can be considered independent of the number of impacts, because only a decrease of 3\% to $6 \%$ was detected between the first and third impact, this variation being smaller than the experimental scatter (9\%). Therefore the agglomerated cork is a good material for applications requiring good performance against multi-impacts.

\section{Conclusions}

In this work, the multi-impact behaviour of agglomerated-cork specimens was studied experimentally. Consecutive impacts for two impact energies were performed on specimens with three different thicknesses. Maximum contact force, maximum strain, and absorbed energy were analysed.

The maximum contact force and maximum strain decreased with increasing specimen thickness for both impact energies studied. The behaviour of these variables when subjected to consecutive impacts was related to the load-strain curve of agglomerated cork under compression, as impact energies exerting strains in the plateau region of the curve led to almost constant maximum contact forces. Only for impact energies that cause strain levels located in the densification stage of the load-strain curve, did the maximum force increase with each impact event. By contrast, the maximum strain increased with each consecutive impact test when the impact energy level caused strains located in the plateau region, and remained almost constant in the densification stage.

A high percentage (70-80\%) of the impact energy was absorbed for both impact energies studied. The absorbed-energy percentage increased slightly at higher impact-energy levels. As a result, agglomerated cork shows excellent energy-absorption capabilities, since the absorbed energy can be considered independent of the number of impacts.

\section{References}

[1] I. Alcântara, F. Teixeira-Dias, M. Paulino, Cork composites for the absorption of impact energy, Compos. Struct. 95 (2013) 16-27.

[2] P. Viot, Polymer foams to optimize passive safety structures in helmets, Int. J. Crashworthiness 12 (3) (2007) 299-310.

[3] Q. Fang, F.Q. Yang, M. Meng, Z.W. Wang, Experiment and research of bumper with double layer inflating safe cushion, Appl. Mech. Mater. 536-537 (2014) 1378-1383. 
[4] M.I. Thiyahuddin, Y.T. Gu, D.P. Thambiratnam, H.M. Thilakarathna, Impact and energy absorption of portable water-filled road safety barrier system fitted with foam, Int. J. Impact Eng. 72 (2014) 26-39.

[5] I. Ivañez, C. Santiuste, S. Sanchez-Saez, FEM analysis of dynamic flexural behaviour of composite sandwich beams with foam core, Compos. Struct. 92 (9) (2010) 2285-2291.

[6] U.E. Ozturk, G. Anlas, Finite element analysis of expanded polystyrene foam under multiple compressive loading and unloading, Mater. Des. 32 (2011) 773-780.

[7] S. Petit, C. Bouvet, A. Bergerot, J.-J. Barrau, Impact and compression after impact experimental study of composite laminate with a cork thermal shield, Compos. Sci. Technol. 67 (2007) 3286-3299.
[8] O. Castro, J.M. Silva, T. Devezas, A. Silva, L. Gil, Cork agglomerates as an ideal core material in lightweight structures, Mater. Des. 31 (2010) 425-432.

[9] F.A.O. Fernandes, R.J.S. Pascoal, R.J. Alves de Sousa, Modelling impact response of agglomerated cork, Mater. Des. 58 (2014) 499-507.

[10] S. Sanchez-Saez, S.K. García-Castillo, E. Barbero, J. Cirne, Dynamic crushing behaviour of agglomerated cork, Mater. Des. 65 (2015) 743-748.

[11] S. Sanchez-Saez, E. Barbero, J. Cirne, Experimental study of agglomerated-cork cored structures subjected to ballistic impacts, Mater. Lett. 65 (2011) 2152-2154.

[12] R.M. Coelho, R.J. Alves de Sousa, F.A.O. Fernandes, F. Teixeira-Dias, New composite liners for energy absorption purposes, Mater. Des. 43 (2013) 384-392. 